\title{
COHERENT PAIR PRODUCTION IN DEFORMED CRYSTALS WITH A COMPLEX BASE
}

\author{
A. R. MKRTCHYAN, A. A. SAHARIAN*and V. V. PARAZIAN \\ Institute of Applied Problems in Physics, 25 Nersessian Str., 375014 Yerevan, Armenia
}

August 13, 2018

\begin{abstract}
We investigate the coherent electron-positron pair creation by high-energy photons in a periodically deformed single crystal with a complex base. The formula for the corresponding differential cross-section is derived for an arbitrary deformation field. The conditions are specified under which the influence of the deformation is considerable. The case is considered in detail when the photon enters into the crystal at small angles with respect to a crystallographic axis. The results of the numerical calculations are presented for $\mathrm{SiO}_{2}$ single crystal and Moliere parametrization of the screened atomic potentials in the case of the deformation field generated by the acoustic wave of $S$ type. In dependence of the parameters, the presence of deformation can either enhance or reduce the pair creation cross-section. This can be used to control the parameters of the positron sources for storage rings and colliders.
\end{abstract}

Keywords: Interaction of particles with matter; coherent pair production; physical effects of ultrasonics.

PACS Nos.: 41.60.-m, 78.90.+t, 43.35.+d, 12.20.Ds

\section{Introduction}

The investigation of high-energy electromagnetic processes in crystals is of interest not only from the viewpoint of underlying physics but also from the viewpoint of practical applications. If the formation length exceeds the interatomic spacing, the interference effects from all atoms within this length are important and the cross-sections of the electromagnetic processes in crystals can change essentially compared with the corresponding quantities for a single atom (see, for instance, [1-3] and references therein). From the point of view of controlling the parameters of various processes in a medium, it is of interest to investigate the influence of external fields, such as acoustic waves, temperature gradient etc., on the corresponding characteristics. The considerations of concrete processes, such as diffraction radiation [4], transition radiation [5], parametric X-radiation [6], channelling radiation [7, bremsstrahlung by high-energy electrons [8], have shown that the external fields can essentially change the angular-frequency characteristics of the radiation intensities. Motivated by the fact that the basic source for the creation of positrons for high-energy electron-positron colliders is the electron-positron pair creation by

${ }^{*}$ E-mail: saharyan@server.physdep.r.am 
high-energy photons, in [9] we have investigated the influence of the hypersonic wave excited in a crystal on this process. The case of simplest crystal with one atom in the lattice base and the sinusoidal deformation field generated by the hypersound were considered. To have an essential influence of the acoustic wave high-frequency hypersound is needed. Usually this type of waves is excited by high-frequency electromagnetic field through the piezoelectric effect in crystals with a complex base. In the present paper we generalize the results of 9 for crystals with a complex base and for acoustic waves with an arbitrary profile. The numerical calculations are carried out for the quartz single crystal and for the photons of energy $100 \mathrm{GeV}$. The results of the numerical calculations on the base of the formulae given in this paper for the pair creation cross-section by the photons of energy $3.5 \mathrm{GeV}$ are presented in our recent paper [10], where the scheme of experimental setup is proposed for the corresponding measurements on the photon channel of the Yerevan synchrotron.

The paper is organized as follows. In the next section we derive the general formula for the coherent part of the pair creation cross-section averaged on thermal fluctuations and the conditions are specified under which the influence of the deformation field can be considerable. The analysis of the general formula in the cases when the photon enters into the crystal at small angles with respect to crystallographic axes or planes is given in Sec. 3. The results of the numerical calculations for the cross-section as a function of the positron energy and the amplitude of the external excitation are presented. Sec. 4 summarizes the main results of the paper.

\section{Cross-section for the coherent pair creation}

Consider the creation of electron-positron pairs by high-energy photons in a crystal. We denote by $(\omega, \mathbf{k}),\left(E_{+}, \mathbf{p}_{+}\right)$, and $\left(E_{-}, \mathbf{p}_{-}\right)$the energies and momenta for the photon, positron, and electron respectively. In the discussion below the collective index $n$ enumerates the elementary cell and the superscript $j$ enumerates the atoms in a given cell of a crystal. Let $d^{4} \sigma_{0} / d E_{+} d^{3} q=$ $\left|u_{\mathbf{q}}^{(j)}\right|^{2} \sigma_{0}(\mathbf{q})$ be the cross-section for the electron-positron pair creation on an individual $j$-th atom as a function of momentum transfer $\mathbf{q}=\mathbf{k}-\mathbf{p}_{+}-\mathbf{p}_{-}$and $u_{\mathbf{q}}^{(j)}$ is the Fourier transform of the potential for the atom. Usually one writes the quantity $u_{\mathbf{q}}^{(j)}$ in the form $4 \pi Z_{j} e^{2}\left[1-F^{(j)}(q)\right] / q^{2}$, where $Z_{j}$ and $F^{(j)}(q)$ are the number of electrons and the atomic form-factor for the $j$-th atom. In the numerical calculations below we will use the Moliere parametrization of the screened atomic potential. The differential cross-section for the pair creation in a crystal can be written in the form (see, for example, 1, 2] )

$$
\sigma(\mathbf{q}) \equiv \frac{d^{4} \sigma}{d E_{+} d^{3} q}=\left|\sum_{n, j} u_{\mathbf{q}}^{(j)} e^{i \mathbf{q} \mathbf{r}_{n}^{(j)}}\right|^{2} \sigma_{0}(\mathbf{q}),
$$

where $\mathbf{r}_{n}^{(j)}$ is the position of an atom in the crystal. The interference factor in Eq. (11) is responsible for coherent effects arising due to periodical arrangement of the atoms in a crystal. At nonzero temperature one has $\mathbf{r}_{n}^{(j)}=\mathbf{r}_{n 0}^{(j)}+\mathbf{u}_{t n}^{(j)}$, where $\mathbf{u}_{t n}^{(j)}$ is the displacement of $j$-th atom with respect to the equilibrium positions $\mathbf{r}_{n 0}^{(j)}$ due to the thermal vibrations. After averaging on thermal fluctuations, the cross-section is written in the form (see, for instance, [1] for the case 
of a crystal with a simple cell)

$$
\sigma(\mathbf{q})=\left\{N \sum_{j}\left|u_{\mathbf{q}}^{(j)}\right|^{2}\left(1-e^{-q^{2} \overline{u_{t}^{(j) 2}}}\right)+\left|\sum_{n, j} u_{\mathbf{q}}^{(j)} e^{i \mathbf{q} \mathbf{r}_{n 0}^{(j)}} e^{-\frac{1}{2} q^{2} \overline{u_{t}^{(j) 2}}}\right|^{2}\right\} \sigma_{0}(\mathbf{q})
$$

where $N$ is the number of cells, $\overline{u_{t}^{(j) 2}}$ is the temperature dependent mean-squared amplitude of the thermal vibrations of the $j$-th atom, $e^{-q^{2} \overline{u_{t}^{(j) 2}}}$ is the corresponding Debye-Waller factor. In formula (2) the first term in figure braces does not depend on the direction of the vector $\mathbf{k}$ and determines the contribution of incoherent effects. The contribution of coherent effects is presented by the second term. By taking into account the formula for the cross-section on a single atom (see, e.g., [1, 2]), in the region of transferred momenta $q \ll m_{e}$ the corresponding part of the cross-section in a crystal can be presented in the form (the system of units $\hbar=c=1$ is used)

$$
\sigma_{c}=\frac{e^{2}}{(2 \pi)^{3} \omega^{2}} \frac{q_{\perp}^{2}}{q_{\|}^{2}}\left(\frac{\omega \delta}{m_{e}^{2}}-1+\frac{2 \delta}{q_{\|}}-\frac{2 \delta^{2}}{q_{\|}^{2}}\right)\left|\sum_{n, j} u_{\mathbf{q}}^{(j)} e^{i \mathbf{q r}_{n 0}^{(j)}} e^{-\frac{1}{2} q^{2} \overline{u_{t}^{(j) 2}}}\right|^{2}
$$

where $\mathbf{q}_{\|}$and $\mathbf{q}_{\perp}$ are the components of the vector $\mathbf{q}$ parallel and perpendicular to the direction of the photon momentum $\mathbf{k}, \delta=1 / l_{c}$ is the minimum longitudinal momentum transfer, and $l_{c}=2 E_{+} E_{-} /\left(m_{e}^{2} \omega\right)$ is the formation length for the pair creation process.

When external influences are present (for example, in the form of acoustic waves) the positions of atoms in the crystal can be written as $\mathbf{r}_{n 0}^{(j)}=\mathbf{r}_{n e}^{(j)}+\mathbf{u}_{n}^{(j)}$, where $\mathbf{r}_{n e}^{(j)}$ determines the equilibrium position of an atom in the situation without the deformation, $\mathbf{u}_{n}^{(j)}$ is the displacement of the atom caused by the external influence. We will consider deformations with the periodical structure:

$$
\mathbf{u}_{n}^{(j)}=\mathbf{u}_{0} f\left(\mathbf{k}_{s} \mathbf{r}_{n e}^{(j)}\right),
$$

where $\mathbf{u}_{0}$ and $\mathbf{k}_{s}$ are the amplitude and wave vector corresponding to the deformation field, $f(x)$ is an arbitrary function with the period $2 \pi, \max f(x)=1$. In the discussion below we will assume that $f(x) \in C^{\infty}(R)$. Note that the dependence of $\mathbf{u}_{n}^{(j)}$ on the time coordinate for the case of acoustic waves we can disregard, as for particle energies we are interested in, the characteristic time for the change of the deformation field is much greater compared with the passage time of particles through the crystal. For the deformation field given by Eq. (44) the sum over $n$ in (2) can be transformed into the form

$$
\sum_{n} u_{\mathbf{q}}^{(j)} e^{i \mathbf{q r}_{n 0}^{(j)}}=\sum_{m=-\infty}^{\infty} F_{m}\left(\mathbf{q} \mathbf{u}_{0}\right) \sum_{n} u_{\mathbf{q}}^{(j)} e^{i \mathbf{q}_{m} \mathbf{r}_{n e}^{(j)}}
$$

where $\mathbf{q}_{m}=\mathbf{q}+m \mathbf{k}_{s}$ and $F_{m}(x)$ is the Fourier-transform of the function $e^{i x f(t)}$ :

$$
F_{m}(x)=\frac{1}{2 \pi} \int_{-\pi}^{\pi} e^{i x f(t)-i m t} d t
$$

Below we need to have the asymptotic behavior of this function for large values $m$. For a fixed $x$ and under the assumptions for the function $f(x)$ given above, by making use the stationary phase method we can see that $F_{m}(x) \sim \mathcal{O}\left(|m|^{-\infty}\right)$ for $m \rightarrow \infty$.

For a lattice with a complex cell the coordinates of the atoms can be presented in the form $\mathbf{r}_{n e}^{(j)}=\mathbf{R}_{n}+\rho^{(j)}$, where $\mathbf{R}_{n}$ determines the positions of the atoms for one of primitive lattices, 
and $\rho^{(j)}$ are the equilibrium positions for other atoms inside $n$-th elementary cell with respect to $\mathbf{R}_{n}$. By taking into account this, one obtains

$$
\sum_{m=-\infty}^{\infty} F_{m}\left(\mathbf{q u} \mathbf{u}_{0}\right) \sum_{j, n} u_{\mathbf{q}}^{(j)} e^{-\frac{1}{2} q^{2} \overline{u_{t}^{(j) 2}}} e^{i \mathbf{q}_{m} \mathbf{r}_{n e}^{(j)}}=\sum_{m=-\infty}^{\infty} F_{m}\left(\mathbf{q u} \mathbf{u}_{0}\right) S\left(\mathbf{q}, \mathbf{q}_{m}\right) \sum_{n} e^{i \mathbf{q}_{m} \mathbf{R}_{n}},
$$

where

$$
S\left(\mathbf{q}, \mathbf{q}_{m}\right)=\sum_{j} u_{\mathbf{q}}^{(j)} e^{i \mathbf{q}_{m} \rho^{(j)}} e^{-\frac{1}{2} q^{2} \overline{u_{t}^{(j) 2}}}
$$

is the factor determined by the structure of the elementary cell. For thick crystals the sum over cells in (7) can be presented as a sum over the reciprocal lattice:

$$
\sum_{n} e^{i \mathbf{q}_{m} \mathbf{R}_{n}}=\frac{(2 \pi)^{3}}{\Delta} \sum_{\mathbf{g}} \delta\left(\mathbf{q}-\mathbf{g}_{m}\right), \mathbf{g}_{m}=\mathbf{g}-m \mathbf{k}_{s}
$$

where $\Delta$ is the unit cell volume, and $\mathbf{g}$ is the reciprocal lattice vector. Due to the $\delta$-function in this formula, the corresponding momentum conservation is written in the form

$$
\mathbf{k}=\mathbf{p}_{+}+\mathbf{p}_{-}+\mathbf{g}-m \mathbf{k}_{s}
$$

where $-m \mathbf{k}_{s}$ stands for the momentum transfer to the external field. As the main contribution into the coherent part of the cross-section comes from the longitudinal momentum transfer of an order $\delta$, the influence of the external excitation may be considerable if $|m| k_{s}$ is of an order $\delta$. The corresponding condition will be specified below. Another consequence of the $\delta$-function in (9) is that the function (6) enters into the cross-section in the form $F_{m}\left(\mathbf{g}_{m} \mathbf{u}_{0}\right)$. Now it can be seen that in the sum over $m$ in (7) the main contribution comes from the terms for which $\left|m \mathbf{k}_{s} \mathbf{u}_{0}\right| \lesssim\left|\mathbf{g u}_{0}\right|$, or equivalently $|m| \lesssim \lambda_{s} / a$, where $\lambda_{s}=2 \pi / k_{s}$ is the wavelength of the external excitation, and $a$ is of the order of the lattice spacing. Indeed, for the terms with $\left|m \mathbf{k}_{s} \mathbf{u}_{0}\right| \gg\left|\mathbf{g} \mathbf{u}_{0}\right|$ one has $F_{m}\left(\mathbf{g}_{m} \mathbf{u}_{0}\right) \approx F_{m}\left(m \mathbf{k}_{s} \mathbf{u}_{0}\right)$, and the phase of the integrand in (6) is equal to $m\left[\mathbf{k}_{s} \mathbf{u}_{0} f(t)-t\right]$. Under the condition $\left|\mathbf{k}_{s} \mathbf{u}_{0} f^{\prime}(t)\right|<1$ this phase has no stationary point and one has $F_{m}\left(m \mathbf{k}_{s} \mathbf{u}_{0}\right)=\mathcal{O}\left(|m|^{-\infty}\right), m \rightarrow \infty$ and the corresponding contribution is strongly suppressed. By taking into account that for practically important cases one has $\mathbf{k}_{s} \mathbf{u}_{0} \sim u_{0} / \lambda_{s} \ll 1$, we see that the assumption made means that the derivative $f^{\prime}(t)$ is not too large. By making use of the formulae given above, the square of the modulus for the sum (5) takes the form

$$
\begin{aligned}
\mid \sum_{j, n} u_{\mathbf{q}}^{(j)} e^{i \mathbf{q} \mathbf{r}_{n 0}} e^{-\left.\frac{1}{2} q^{2} \overline{u_{t}^{(j) 2}}\right|^{2}=} & \sum_{m, \mathbf{g}} F_{m}\left(\mathbf{g}_{m} \mathbf{u}_{0}\right) S\left(\mathbf{g}_{m}, \mathbf{g}\right) \delta\left(\mathbf{q}-\mathbf{g}_{m}\right) \\
& \times \sum_{m^{\prime}} F_{m^{\prime}}^{*}\left(\mathbf{q} \mathbf{u}_{0}\right) S^{*}\left(\mathbf{q}, \mathbf{q}_{m^{\prime}}\right) \sum_{n} e^{i\left(m-m^{\prime}\right) \mathbf{k}_{s} \mathbf{R}_{n}} .
\end{aligned}
$$

In the case $m^{\prime} \neq m$ the main contribution into the sum over $n$ comes from the summands satisfying the condition $\left(m^{\prime}-m\right) \mathbf{k}_{s}=\mathbf{g}$. It follows from here that $\left|m^{\prime}-m\right| \gtrsim \lambda_{s} / a$. By taking into account that the main contribution into the sum over $m$ comes from $|m| \lesssim \lambda_{s} / a$, we conclude that $\left|m^{\prime}\right| \gtrsim \lambda_{s} / a \gg 1$. Now we see that for the function $F_{m^{\prime}}\left(\mathbf{q u}_{0}\right)$ the ratio of the order to the argument is estimated as $m^{\prime} / \mathbf{q u} \mathbf{u}_{0} \sim \lambda_{s} / 2 \pi u_{0}$. Combining this with the asymptotic behavior of the function $F_{m}(x)$ for large values of the order given in the paragraph after formula (6) $)$, we see that under the condition $u_{0} / \lambda_{s} \ll 1$ the contribution of the terms with $m \neq m^{\prime}$ in the sum (111) 
is small compared to the diagonal terms. In the case $m=m^{\prime}$ the sum over $n$ in the left hand side is equal to the number of cells, $N$, in a crystal and the square of the modulus for the sum on the left of Eq. (11) can be written as

$$
\left|\sum_{j, n} u_{\mathbf{q}}^{(j)} e^{i \mathbf{q} \mathbf{r}_{n 0}} e^{-\frac{1}{2} q^{2} \overline{u_{t}^{(j) 2}}}\right|^{2}=N \frac{(2 \pi)^{3}}{\Delta} \sum_{m, \mathbf{g}}\left|F_{m}\left(\mathbf{g}_{m} \mathbf{u}_{0}\right)\right|^{2}\left|S\left(\mathbf{g}_{m}, \mathbf{g}\right)\right|^{2} .
$$

Substituting this expression into formula (3) and integrating over the vector $\mathbf{q}$ by using the $\delta$-function, for the cross-section one obtains

$$
d \sigma=\int \sigma(\mathbf{q}) d^{3} q=N_{0}\left(d \sigma_{n}+d \sigma_{c}\right)
$$

with $d \sigma_{n}$ and $d \sigma_{c}$ being the incoherent and coherent parts of the cross-section per atom and $N_{0}$ is the number of atoms in the crystal. The coherent part of the cross-section is determined by the formula

$$
\frac{d \sigma_{c}}{d E_{+}}=\frac{e^{2} N}{\omega^{2} N_{0} \Delta} \sum_{m, \mathbf{g}} \frac{g_{m \perp}^{2}}{g_{m \|}^{2}}\left(\frac{\omega^{2}}{2 E_{+} E_{-}}-1+\frac{2 \delta}{g_{m \|}}-\frac{2 \delta^{2}}{g_{m \|}^{2}}\right)\left|F_{m}\left(\mathbf{g}_{m} \mathbf{u}_{0}\right)\right|^{2}\left|S\left(\mathbf{g}_{m}, \mathbf{g}\right)\right|^{2},
$$

where the vector $\mathbf{g}_{m}$ is defined by relation (9) and the summation goes under the constraint $g_{m \|} \geq \delta$. For a simplest crystal with one atom in the lattice base and sinusoidal deformation field, $f(z)=\sin \left(z+\varphi_{0}\right)$, the formula (14) reduces to the result obtained in [9]. Note that for this type of deformation one has the Fourier-transform

$$
F_{m}(x)=e^{i m \varphi_{0}} J_{m}(x),
$$

with the Bessel function $J_{m}(x)$.

The formula for the pair creation in an undeformed crystal is obtained from (14) taking $\mathbf{u}_{0}=0$. In this limit the contribution of the term with $m=0$ remains only with $F_{0}(0)=1$. The corresponding formula for a crystal with simple lattice base can be found, for instance, in [1, 2]. Now we see that formula (14) differs from the formula in an undeformed crystal by the replacement $\mathbf{g} \rightarrow \mathbf{g}_{m}$, and by an additional summation over $m$ with weights $\left|F_{m}\left(\mathbf{g}_{m} \mathbf{u}_{0}\right)\right|^{2}$. This corresponds to the presence of an additional one-dimensional superlattice with the period $\lambda_{s}$ and the reciprocal lattice vector $m \mathbf{k}_{s}, m=0, \pm 1, \pm 2, \ldots$ As the main contribution into the cross-section comes from the terms with $g_{m \|} \sim \delta$, the influence of the deformation field may be considerable if $\left|m k_{s \|}\right| \gtrsim \delta$. Combining this with the previous estimates we find the following condition: $u_{0} / \lambda_{s} \gtrsim a / 4 \pi^{2} l_{c}$. At high energies one has $a / l_{c} \ll 1$ and this condition can be consistent with the condition $u_{0} / \lambda_{s} \ll 1$.

\section{$3 \quad$ Limiting cases and numerical results}

If the photon moves in a non-oriented crystal, in formula (14) the summation over $\mathbf{g}$ can be replaced by the integration and the pair creation cross-section coincides with that in an amorphous medium. Note that for an amorphous medium the ratio $u_{0} / \lambda_{s}$ can be relatively larger compared with the corresponding quantity in crystals. Below we consider the case when the photon enters into the crystal at small angle $\theta$ with respect to the crystallographic $z$-axis of the orthogonal lattice. The corresponding reciprocal lattice vector components are $g_{i}=2 \pi n_{i} / a_{i}$, 
$n_{i}=0, \pm 1, \pm 2, \ldots$, where $a_{i}, i=1,2,3$, are lattice constants in the corresponding directions. We can write

$$
g_{m \|}=g_{m z} \cos \theta+\left(g_{m x} \sin \alpha+g_{m y} \cos \alpha\right) \sin \theta,
$$

where $\alpha$ is the angle between the projection of the vector $\mathbf{k}$ on the plane $(x, y)$ and $y$-axis. For small angles $\theta$ the main contribution into the cross-section comes from the summands with $g_{z}=0$ and from formula (14) one finds

$$
\frac{d \sigma_{c}}{d E_{+}} \approx \frac{e^{2} N}{\omega^{2} N_{0} \Delta} \sum_{m, g_{x}, g_{y}} \frac{g_{\perp}^{2}}{g_{m \|}^{2}}\left(\frac{\omega^{2}}{2 E_{+} E_{-}}-1+\frac{2 \delta}{g_{m \|}}-\frac{2 \delta^{2}}{g_{m \|}^{2}}\right)\left|F_{m}\left(\mathbf{g}_{m} \mathbf{u}_{0}\right)\right|^{2}\left|S\left(\mathbf{g}_{m}, \mathbf{g}\right)\right|^{2},
$$

where $g_{\perp}^{2}=g_{x}^{2}+g_{y}^{2}$, and the summation goes over the region $g_{m \|} \geq \delta$ with

$$
g_{m \|} \approx-m k_{z}+\left(g_{x} \sin \alpha+g_{y} \cos \alpha\right) \theta .
$$

Note that in the arguments of the functions $F_{m}$ and $S$ we have $\mathbf{g}_{m} \approx\left(g_{x}, g_{y}, 0\right)$.

If the photon moves far from the crystallographic planes (angles $\alpha$ and $\pi / 2-\alpha$ are not small compared with unity), in Eq. (17) the summation over $g_{x}$ and $g_{y}$ can be replaced by the integration, $\sum_{g_{x}, g_{y}} \rightarrow\left(a_{1} a_{2} / 4 \pi^{2}\right) \int d g_{x} d g_{y}$, and one receives

$$
\begin{aligned}
\frac{d \sigma_{c}}{d E_{+}} \approx & \frac{e^{2} N \omega^{-2}}{4 \pi^{2} N_{0} a_{3}} \sum_{m} \int d g_{x} d g_{y} \frac{g_{\perp}^{2}}{g_{m \|}^{2}}\left(\frac{\omega^{2}}{2 E_{+} E_{-}}-1+\frac{2 \delta}{g_{m \|}}-\frac{2 \delta^{2}}{g_{m \|}^{2}}\right) \\
& \times\left|F_{m}\left(\mathbf{g}_{m} \mathbf{u}_{0}\right)\right|^{2}\left|S\left(\mathbf{g}_{m}, \mathbf{g}\right)\right|^{2}
\end{aligned}
$$

where the integration goes under the constraint $g_{m \|} \geq \delta$.

We now assume that the photon enters into the crystal at small angle $\theta$ with respect to the crystallographic axis $z$ and near the crystallographic plane $(y, z)$ (the angle $\alpha$ is small). In this case with the change of $\delta$, the sum over $g_{x}$ and $g_{y}$ will drop sets of terms which leads to the abrupt change of the corresponding cross-section. Two cases have to be distinguished. Under the condition $\delta \sim 2 \pi \theta / a_{2}$, in Eq. (17) for the longitudinal component one has

$$
g_{m \|} \approx-m k_{z}+\theta g_{y} \geq \delta .
$$

In this case the summation over the component $g_{x}$ can be replaced by the integration, $\sum_{g_{x}} \rightarrow$ $\left(a_{1} / 2 \pi\right) \int d g_{x}$, and we have the formula

$$
\begin{aligned}
\frac{d \sigma_{c}}{d E_{+}} \approx & \frac{e^{2} N \omega^{-2}}{2 \pi N_{0} a_{2} a_{3}} \sum_{m, g_{y}} \int d g_{x} \frac{g_{\perp}^{2}}{g_{m \|}^{2}}\left(\frac{\omega^{2}}{2 E_{+} E_{-}}-1+\frac{2 \delta}{g_{m \|}}-\frac{2 \delta^{2}}{g_{m \|}^{2}}\right) \\
& \times\left|F_{m}\left(\mathbf{g}_{m} \mathbf{u}_{0}\right)\right|^{2}\left|S\left(\mathbf{g}_{m}, \mathbf{g}\right)\right|^{2} .
\end{aligned}
$$

This formula can be further simplified under the assumption $\mathbf{u}_{0} \perp \mathbf{a}_{1}$. In this case in the argument of the function $F_{m}$ one has $\mathbf{g}_{m} \mathbf{u}_{0} \approx g_{y} u_{0 y}$ and we obtain the formula

$$
\frac{d \sigma_{c}}{d E_{+}} \approx \frac{e^{2} N \omega^{-2}}{2 \pi N_{0} a_{2} a_{3}} \sum_{m, g_{y}} \frac{\left|F_{m}\left(g_{y} u_{0 y}\right)\right|^{2}}{g_{m \|}^{2}}\left(\frac{\omega^{2}}{2 E_{+} E_{-}}-1+\frac{2 \delta}{g_{m \|}}-\frac{2 \delta^{2}}{g_{m \|}^{2}}\right) \int d g_{x} g_{\perp}^{2}\left|S\left(\mathbf{g}_{m}, \mathbf{g}\right)\right|^{2},
$$

with an effective structure factor determined by the integral on the right. 
In the second case we assume that $\delta \sim 2 \pi \theta \alpha / a_{1}$. Now the main contribution into the sum in Eq. (17) comes from the terms with $g_{y}=0$ and two summations remain: over $m$ and over $n_{1}$. The formula for the cross-section takes the form

$$
\frac{d \sigma_{c}}{d E_{+}} \approx \frac{e^{2} N}{\omega^{2} N_{0} \Delta} \sum_{m, n_{1}} \frac{g_{\perp}^{2}}{g_{m \|}^{2}}\left(\frac{\omega^{2}}{2 E_{+} E_{-}}-1+\frac{2 \delta}{g_{m \|}}-\frac{2 \delta^{2}}{g_{m \|}^{2}}\right)\left|F_{m}\left(\mathbf{g}_{m} \mathbf{u}_{0}\right)\right|^{2}\left|S\left(\mathbf{g}_{m}, \mathbf{g}\right)\right|^{2},
$$

where

$$
g_{m \|} \approx-m k_{z}+\psi g_{x}, \psi=\alpha \theta,
$$

and the summation goes over the values $m$ and $n_{1}$ satisfying the condition $g_{m \|} \geq \delta$.

We have carried out numerical calculations for the pair creation cross-section for various values of parameters in the case of $\mathrm{SiO}_{2}$ single crystal at zero temperature. To deal with an orthogonal lattice, we choose as an elementary cell the cell including 6 atoms of silicon and 12 atoms of oxygen (Shrauf elementary cell [11]). For this choice the $y$ and $z$ axes of the orthogonal coordinate system $(x, y, z)$ coincide with the standard $Y$ and $Z$ axes of the quartz crystal, whereas the angle between the axes $x$ and $X$ is equal to $\pi / 6$. For the potentials of atoms we take Moliere parametrization

$$
u_{\mathbf{q}}^{(j)}=\sum_{i=1}^{3} \frac{4 \pi Z_{j} e^{2} \alpha_{i}}{q^{2}+\left(\chi_{i} / R_{j}\right)^{2}}
$$

where $\alpha_{i}=\{0.1,0.55,0.35\}, \chi_{i}=\{6.0,1.2,0.3\}$, and $R_{j}$ is the screening radius for the $j$-th atom in the elementary cell. The calculations are carried out for the sinusoidal transversal acoustic wave of the $S$ type (the corresponding parameters can be found in Ref. [12]) for which the vector of the amplitude of the displacement is directed along $X$-direction of quartz single crystal, $\mathbf{u}_{0}=\left(u_{0}, 0,0\right)$, and the velocity is $4.687 \cdot 10^{5} \mathrm{~cm} / \mathrm{sec}$. The vector determining the direction of the hypersound propagation lies in the plane $Y Z$ and has the angle with the axis $Z$ equal to $0.295 \mathrm{rad}$. As the axis $z$ we choose the axis $Z$ of the quartz crystal. The corresponding function $F(x)$ is determined by formula (15). Note that in the case of potential (25) for the integral in formula (22) one has

$$
\int d g_{x} g_{\perp}^{2}\left|S\left(\mathbf{g}_{m}, \mathbf{g}\right)\right|^{2} \approx 32 \pi^{3} e^{4} \sum_{j, j^{\prime}, i, i^{\prime}} \cos \left(g_{y} \rho_{y}^{\left(j j^{\prime}\right)}\right) \frac{e^{-\rho_{x}^{\left(j j^{\prime}\right)} \sqrt{g_{y}^{2}+\chi_{i}^{2} / R_{j}^{2}}}}{\sqrt{g_{y}^{2}+\chi_{i}^{2} / R_{j}^{2}}} \frac{\alpha_{i} \alpha_{i^{\prime}} Z_{j} Z_{j^{\prime}} \chi_{i}^{2} / R_{j}^{2}}{\chi_{i}^{2} / R_{j}^{2}-\chi_{i^{\prime}}^{2} / R_{j^{\prime}}^{2}}
$$

where we use the notation $\rho_{q}^{\left(j j^{\prime}\right)}=\left|\rho_{q}^{(j)}-\rho_{q}^{\left(j^{\prime}\right)}\right|, q=x, y$. For the case $\chi_{i} / R_{j}=\chi_{i^{\prime}} / R_{j^{\prime}}$ in (26) one has uncertainty $0 / 0$ which has to be evaluated by the Lopitale rule.

Numerical calculations show that, in dependence of the values for the parameters, the external excitation can either enhance or reduce the cross-section of the pair creation process. As an illustration of the enhancement in figure 1 (left panel) we have depicted the quantity $d \sigma_{c} / d E_{+}$ evaluated by formula (22) with the effective structure factor (26) as a function of the ratio $E_{+} / \omega$ in the case of $\mathrm{SiO}_{2}$ single crystal and Moliere parametrization of the screened atomic potential for $u_{0}=0$ (dashed curve) and $2 \pi u_{0} / a_{2}=2.4$ (full curve). The deformation is induced by the transversal acoustic wave of the $S$ type with frequency $5 \mathrm{MHz}$. The values for the other parameters are as follows: $\theta=0.002 \mathrm{rad}, \omega=100 \mathrm{GeV}$. As the cross-section is symmetric under the replacement $E_{+} / \omega \rightarrow 1-E_{+} / \omega$, we have plotted the graphs for the region $0 \leq E_{+} / \omega \leq 0.5$ only. In figure 1 (right panel) the cross-section evaluated by formula (22) is presented as a function of $2 \pi u_{0} / a_{2}$ for the positron energy corresponding to $E_{+} / \omega=0.5$. The values of the other parameters are the same as those for the left figure. Note that for the chosen values of the parameters 
one has $\lambda_{s} \approx 9.4 \times 10^{-4} \mathrm{~cm}$, whereas $l_{c} \approx 3.8 \times 10^{-6} \mathrm{~cm}$ for the energies $E_{+}=E_{-}=50 \mathrm{Gev}$ and, hence, $\lambda_{s} \gg l_{c}$.
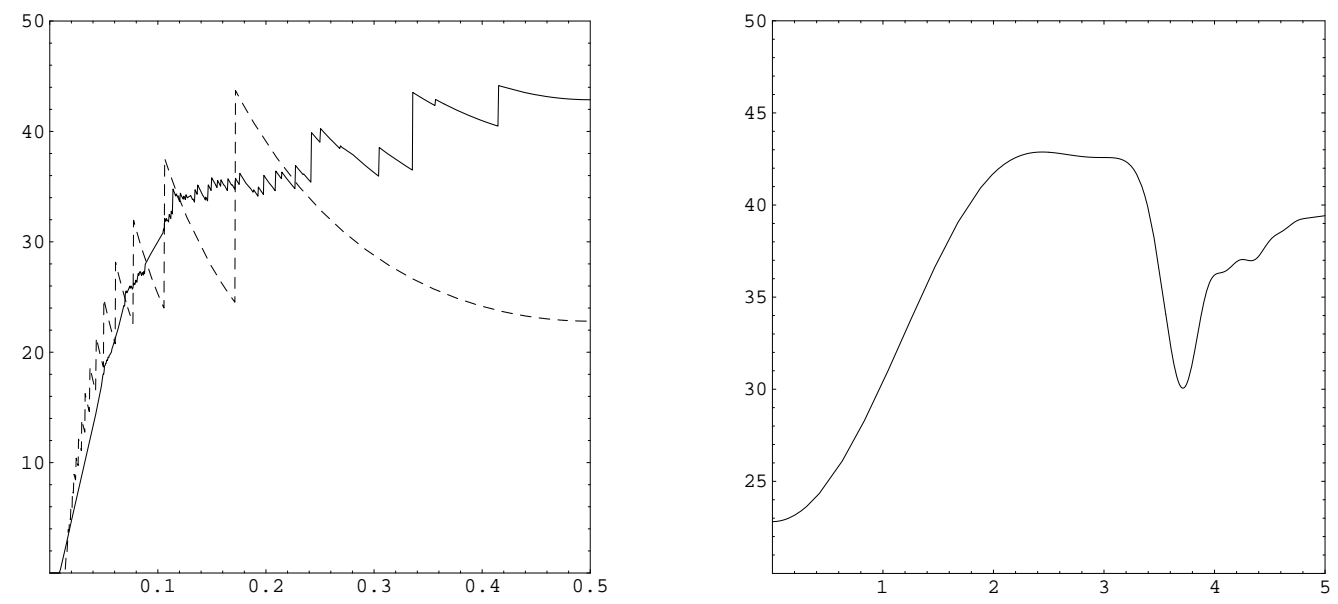

Figure 1: Coherent part of the pair creation cross-section, $10^{-3}\left(m_{e}^{2} \omega / e^{6}\right) d \sigma_{c} / d E_{+}$, evaluated by formula (22) with (26), in the quartz single crystal for the sinusoidal transversal acoustic wave of the $S$ type with frequency $5 \mathrm{GHz}$ as a function of $E_{+} / \omega$ (left panel) for $u_{0}=0$ (dashed curve), $2 \pi u_{0} / a_{2}=2.4$ (full curve) and as a function of $2 \pi u_{0} / a_{2}$ for the positron energy corresponding to $E_{+} / \omega=0.5$. The values for the other parameters are as follows: $\theta=0.002 \mathrm{rad}, \omega=100$ $\mathrm{GeV}$.

In figure 2 (left panel) we have presented the cross-section evaluated by formula (23) as a function of the ratio $E_{+} / \omega$ for $u_{0}=0$ (dashed curve) and $2 \pi u_{0} / a_{1}=2.1$ (full curve) in the case $\psi=0.001$ and for the photon energy $\omega=100 \mathrm{GeV}$. The values for the other parameters are the same as in figure 1. In figure 2 (right panel) we have plotted the cross-section evaluated by formula (23) as a function of $2 \pi u_{0} / a_{1}$ for the positron energy corresponding to $E_{+} / \omega=0.5$ and for $\psi=0.001 \mathrm{rad}$. The values for the other parameters are the same as for the left panel.

\section{Conclusion}

The present paper is devoted to the investigation of the electron-positron pair creation by highenergy photons in a crystal with a complex lattice base in the presence of deformation field of an arbitrary periodic profile. The latter can be induced, for example, by acoustic waves. The influence of the deformation field can serve as a possible mechanism to control the angularenergetic characteristics of the created particles. The importance of this is motivated by that the basic source to creating positrons for high-energy colliders is the electron-positron pair creation by high-energy photons. In a crystal the cross-section is a sum of coherent and incoherent parts. The coherent part of the cross-section per single atom, averaged on thermal fluctuations, is given by formula (14). In this formula the factor $\left|F_{m}\left(\mathbf{g}_{m} \mathbf{u}_{0}\right)\right|^{2}$ is determined by the function describing the displacement of the atoms due to the deformation field, and the factor $\left|S\left(\mathbf{g}_{m}, \mathbf{g}\right)\right|^{2}$ is determined by the structure of the crystal elementary cell. Compared with the cross-section in an undeformed crystal, formula (14) contains an additional summation over the reciprocal lattice vector $m \mathbf{k}_{s}$ of the one-dimensional superlattice induced by the deformation field. We have argued that the influence of the deformation field on the cross-section can be remarkable under the condition $4 \pi^{2} u_{0} / a \gtrsim \lambda_{s} / l_{c}$. Note that for the deformation with $4 \pi^{2} u_{0} / a>1$ this condition 

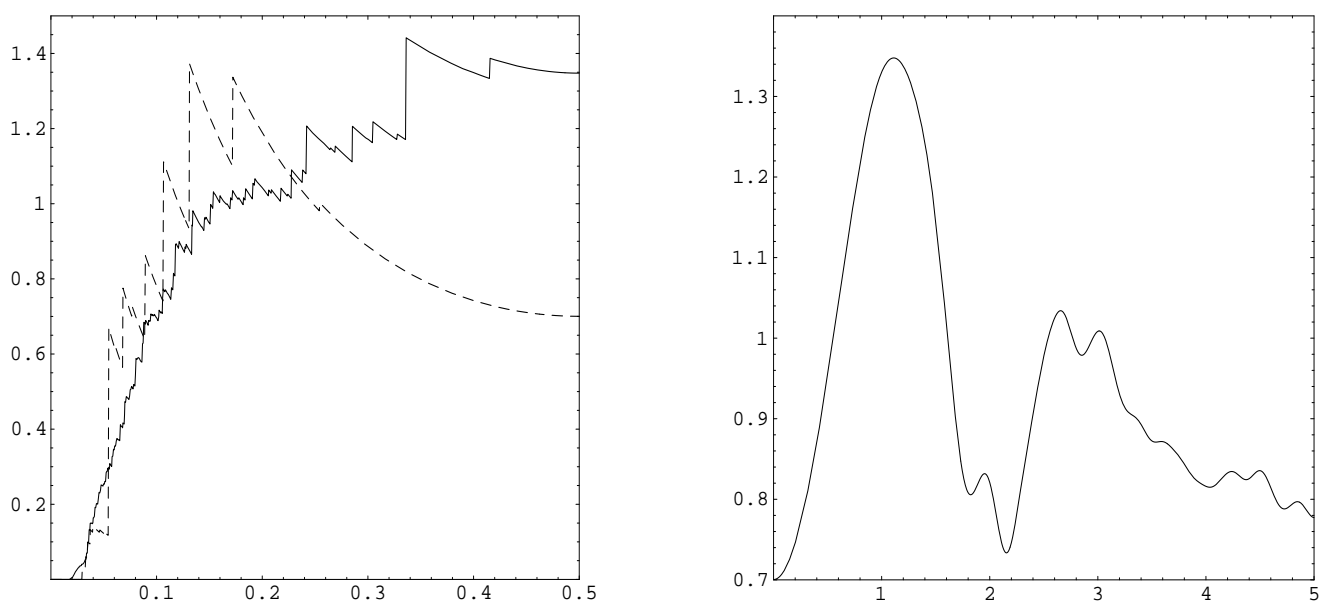

Figure 2: Pair creation cross-section, $10^{-3}\left(m_{e}^{2} \omega / e^{6}\right) d \sigma_{c} / d E_{+}$, evaluated by formula (23), as a function of $E_{+} / \omega$ (left panel) for $u_{0}=0$ (dashed curve), $2 \pi u_{0} / a_{2}=2.1$ (full curve) and as a function of $2 \pi u_{0} / a_{2}$ (right panel) for the positron energy corresponding to $E_{+} / \omega=0.5$. The values for the other parameters are as follows: $\psi=0.001 \mathrm{rad}, \omega=100 \mathrm{GeV}$.

is less restrictive than the naively expected one $\lambda_{s} \lesssim l_{c}$. The role of coherence effects in the pair creation cross-section is essential when the photon enters into the crystal at small angles with respect to a crystallographic axis. In this case the main contribution into the coherent part of the cross-section comes from the crystallographic planes, parallel to the chosen axis (axis $z$ in our consideration). The behavior of this cross-section as a function on the positron energy essentially depends on the angle $\alpha$ between the projection of the photon momentum on the plane $(x, y)$ and $y$-axis. If the photon moves far from the corresponding crystallographic planes, the summation over the perpendicular components of the reciprocal lattice vector can be replaced by the integration and the coherent part of the pair creation cross-section is given by formula (19). When the photon enters into the crystal near a crystallographic plane, two cases have to be distinguished. For the first one $\theta \sim a_{2} / 2 \pi l_{c}$, the summation over $g_{x}$ can be replaced by integration and one obtains formula (21). This formula is further simplified to the form (22) under the assumption $\mathbf{u}_{0} \perp \mathbf{a}_{1}$. In the second case one has $\psi=\alpha \theta \sim a_{1} / 2 \pi l_{c}$, and the main contribution into the cross-section comes from the crystallographic planes parallel to the incidence plane. The corresponding formula for the cross-section takes the form (23). The numerical calculations for the cross-section are carried out in the case of $\mathrm{SiO}_{2}$ single crystal with the Moliere parametrization of the screened atomic potentials and for the deformation field generated by the transversal acoustic wave of $S$ type with frequency $5 \mathrm{GHz}$. Examples of numerical results are depicted in figures 1 and 2. The numerical calculations for various values of the parameters in the problem show that, in dependence of the values for the parameters, the presence of the deformation field can either enhance or reduce the cross-section. This can be used to control the parameters of the positron sources for storage rings and colliders.

\section{Acknowledgment}

We are grateful to Levon Grigoryan and Hrant Khachatryan for valuable discussions and suggestions. The work has been supported by Grant no. 0061 from Ministry of Education and Science of the Republic of Armenia. 


\section{References}

[1] M. L. Ter-Mikaelian, High Energy Electromagnetic Processes in Condensed Media (Wiley Interscience, New York, 1972).

[2] A. I. Akhiezer and N. F. Shulga, High Energy Electrodynamics in Matter (Gordon and Breach, 1996).

[3] V. N. Baier, V. M. Katkov and V. M. Strakhovenko, Electromagnetic Processes at High Energies in Oriented Single Crystals (World Scientific, 1998).

[4] A. R. Mkrtchyan, L. Sh. Grigoryan, A. N. Didenko, A. A. Saharian and A. G. Mkrtchyan, Izv. Akad. Nauk. Arm. SSR. Fiz. 24, 62 (1989); A. R. Mkrtchyan, L. Sh. Grigoryan, A. N. Didenko and A. A. Saharian, Sov. Phys. JTP 61, 21 (1991); A. R. Mkrtchyan, L. Sh. Grigoryan, A. A. Saharian and A. N. Didenko, Acustica 75, 1984 (1991); A. A. Saharian, A. R. Mkrtchyan, L. A. Gevorgian, L. Sh. Grigoryan and B. V. Khachatryan, Nucl. Instr. Meth. B173, 211 (2001).

[5] L. Sh. Grigoryan, A. H. Mkrtchyan and A. A. Saharian, Nucl. Instr. Meth. B145 197 (1998).

[6] A. R. Mkrtchyan, H. A. Aslanyan, A. H. Mkrtchyan and R. A. Gasparyan, Phys. Lett. A152, 297 (1991).

[7] A. R. Mkrtchyan, R. A. Gasparyan and R. G. Gabrielyan, Phys. Lett. A115, 410 (1986); JETP 93432 (1987); Phys. Lett. A126, 528 (1988); L. Sh. Grigoryan et al., Nucl. Instr. Meth. B173, 13; 184 (2001); L. Sh. Grigoryan, A. H. Mkrtchyan, H. F. Khachatryan, V. U. Tonoyan and W. Wagner, Nucl. Instr. Meth. B201, 25 (2003).

[8] A. A. Saharian, A. R. Mkrtchyan, V. V. Parazian and L. Sh. Grigoryan, Mod. Phys. Lett. A19, 99 (2004).

[9] A. R. Mkrtchyan, A. A. Saharian, L. Sh. Grigoryan and B. V. Khachatryan, Mod. Phys. Lett. A17, 2571 (2002).

[10] A. R. Mkrtchyan, A. A. Saharian, V. V. Parazian, A. H. Mkrtchyan, H. A. Vardapetyan, A. M. Sirunyan, G. G. Hakobyan and J. V. Manukyan, J. Contemp. Phys. (in press).

[11] J. S. Dana, E. S. Dana and C. Frondel, The System of Mineralogy. Vol. 3 Silica Minerals (John Wiley and Sons, 1962).

[12] Acoustic Crystals, Edited by M. P. Shaskolskaya (Nauka, 1982) (in Russian). 